УСКОРЕННАЯ ПРЕДОПЕРАЦИОННАЯ ПОДГОТОВКА В СОСТАВЕ КОМПЛЕКСНОЙ ПЕРИОПЕРАЦИОННОЙ РЕАБИЛИТАЦИИ ПАЦИЕНТОВ СО СВЕРХОЖИРЕНИЕМ И СИНДРОМОМ ОБСТРУКТИВНОГО АПНОЭ (КЛИНИЧЕСКОЕ НАБЛЮДЕНИЕ)

\author{
() Е.С. Орловская ${ }^{1,2 *}$, Е.Е. Ачкасов ${ }^{2}$, Ю.И. Яшков ${ }^{3}$, Е.А. Зорин ${ }^{1}$, Н.А. Ермаков ${ }^{1}$
}

${ }^{1}$ Национальный медицинский исследовательский центр «Лечебно-реабилитационный центр», Москва, Россия Первый Московский государственный медицинский университет им. И.М. Сеченова (Сеченовский Университет), Москва, Россия

${ }^{3}$ Центр эндохирургии и литотрипсии, Москва, Россия

Ожирение - глобальная социально-экономическая проблема. Наиболее эффективный метод лечения ожирения бариатрическая хирургия. Представленный клинический случай демонстрирует тактику ускоренной периоперационной реабилитации пациента со сверхожирением в сочетании с синдромом обструктивного апноэ сна тяжелой степени. Пациентка 54 лет госпитализирована с жалобами на избыточный вес, невозможность стойкого снижения веса консервативно, выраженную дневную сонливость, частые ночные пробуждения (до 8 раз за ночь). При поступлении масса тела (МТ) пациентки составляла 230 кг при росте 157 см (ИМТ 93,5 кг/м²). При обследовании выявлены синдром апноэ сна смешанного генеза крайне тяжелой степени, хроническая ночная гипоксемия крайне тяжелой степени. Проведена предоперационная подготовка по программе ускоренной периоперационной реабилитации. Длительность предоперационной подготовки составила 19 сут; достигнуто снижение веса на 40 кг (17,4\% потери массы тела), компенсация коморбидных состояний. Пациентка оперирована в объеме лапароскопической рукавной резекции желудка. Течение послеоперационного периода без осложнений. Выписка из стационара на 6-е сутки. При контрольном обследовании через 1 год снижение веса до 153 кг (33,5\% потери массы тела), значимое улучшение качества жизни.

Программа ускоренной периоперационной реабилитации может быть использована как альтернатива стандартной программе реабилитации с имплантацией интрагастрального баллона у пациентов с морбидным ожирением в сочетании с синдромом обструктивного апноэ сна тяжелой степени. Применение данной методики позволяет повысить эффективность лечения данной категории пациентов, а также снизить риск развития периоперационных осложнений

КЛЮЧЕВЫЕ СЛОВА: сверхожирение; бариатрическая хирургия; клинический случай; синдром обструктивного апноэ сна; периоперачионное ведение; СРАР-терапия; ускоренная послеоперационная реабилитация.

\title{
ENCHANCED PREOPERATIVE REHABILITATION AS PART OF THE COMPLEX PERIOPERATIVE REHABILITATION OF PATIENTS WITH SUPER-OBESITY AND OBSTRUCTIVE SLEEP APNEA SYNDROME (CLINICAL OUTLOOK)
}

\author{
(c) Ekaterina S. Orlovskaya ${ }^{1,2 *}$, Evgeniy E. Achkasov², Yury I. Yashkov³, Evgeniy A. Zorin', Nikolay A. Ermakov ${ }^{1}$
}

${ }^{1}$ Medical Rehabilitation Centre, Moscow, Russia.

2I.M. Sechenov First Moscow State Medical University (Sechenov University), Moscow, Russia

${ }^{3}$ Center of Endosurgery and Lithotripsy, Moscow, Russia

Obesity is a global social and economic problem. The bariatric surgery is a most effective treatment for obesity. The presented clinical case demonstrates the usage of principles of enchanced perioperative rehabilitation for the preoperative preparation of a patient with super obesity and with severe obstructive sleep apnea and alveolar hypoventilation syndrome. A 54-year-old patient was hospitalized with complaints of obesity, impossibility of persistent weight loss conservatively, severe daytime sleepiness, frequent nocturnal awakenings (up to 8 times per night). The patient's weight was $230 \mathrm{~kg}$ with a height of $157 \mathrm{~cm}$ $\left(B M I 93.5 \mathrm{~kg} / \mathrm{m}^{2}\right)$. The examination revealed a syndrome of sleep apnea of mixed genesis of extremely severe degree, chronic night hypoxemia of an extremely severe degree. Preoperative preparation was performed in accordance with the program of enchanced perioperative rehabilitation. The duration of preoperative preparation was 19 days; weight loss - $40 \mathrm{~kg}(\% \mathrm{WL}-17,4)$, compensation of comorbidities was achieved as well. After that the patient underwent a laparoscopic sleeve gastrectomy. There were no complications in the postoperative period. Length of postoperative hospital stay was 6 days. At follow-up examination one year after surgery, body weight dropped from 230 to $153 \mathrm{~kg}$ (\%WL-33.5), a significant improvement of the quality of life was achieved.

The enchanced perioperative rehabilitation program can be successfully used as an effective method for preoperartive preparation of the patients with morbid obesity in combination with severe obstructive sleep apnea syndrome.and obesity hypoventilation. It can be a reasonable alternative to the standard program with preoperative intragastric balloon treatment. The use of this technique allows to increase the effectiveness of treatment of these high-risk patients, as well as to reduce the risk of perioperative complications.

KEYWORDS: super-obesity; bariatric surgery; case report; obstructive sleep apnea syndrome; perioperative care; CPAP-therapy. 


\section{АКТУАЛЬНОСТЬ}

В настоящее время ожирение является глобальной социально-экономической проблемой, распространенность которой приобретает масштабы пандемии. Неуправляемо растущая заболеваемость ожирением привлекает внимание всего мира [1, 2].

Одним из самых эффективных методов лечения ожирения является бариатрическая хирургия [3, 4]. В последние годы все большее внимание уделяется вопросам периоперационного ведения пациентов с ожирением. В связи с ежегодно возрастающим числом выполняемых бариатрических операций возникла необходимость в поиске и реализации новых стратегий, направленных на оптимизацию пери- и послеоперационного ведения пациентов с избыточным весом; методом достижения данной цели является внедрение программы ускоренного восстановления после операции (ERAS) в бариатрической хирургии [5-8]. В данных протоколах большое внимание уделяется вопросам интра- и послеоперационного ведения бариатрических пациентов, в то время как многие вопросы предоперационной подготовки и компенсации общесоматической патологии остаются дискутабельными. В частности, это касается периоперационного ведения пациентов с морбидным ожирением в сочетании с синдромом обструктивного апноэ сна (СОАС).

Хирургические пациенты СОАС имеют более высокий риск развития осложнений [9]. На фоне гипоксемии отмечается повышение риска раневых осложнений, несостоятельности анастомозов, тромбоэмболических осложнений, ишемии миокарда, нарушений ритма сердца в раннем послеоперационном периоде. При этом у пациентов с ожирением количество осложнений всегда выше.

С учетом ряда особенностей данной категории пациентов, в том числе мнестико-интеллектуальных расстройств на фоне хронической гипоксемии, крайне низкой, по данным ряда авторов, комплаентности пациентов к продолжительному лечению в целом и СРАР-терапии (Continious Positive Airway Pressure - методики создания постоянного положительного давления в дыхательных путях) в частности, выявляется необходимость оптими- зации тактики периоперационной реабилитации этой группы пациентов $[10,11]$.

\section{ОПИСАНИЕ СЛУЧАЯ}

Пациентка 54 лет, пенсионерка, была госпитализирована в хирургическое отделение в мае 2019 г. с жалобами на избыточный вес, невозможность стойкого снижения веса консервативно, боли в суставах при физической нагрузке, одышку при физической нагрузке и в покое, выраженную дневную сонливость, частые ночные пробуждения (до 8 раз за ночь).

Пациентка более 25 лет страдала избыточным весом. Многочисленные попытки снижения веса стойкого эффекта не приносили. После выхода на пенсию в течение 4 лет прибавка в весе составила 60 кг. В течение 1 года отмечала появление выраженной дневной сонливости, частых ночных пробуждений из-за ощущения удушья. Была госпитализирована для комплексного обследования и подготовки к бариатрической операции.

При поступлении масса тела (МТ) пациентки составила 230 кг при росте 157 см (индекс массы тела (ИМТ) 93,5 кг/м²), отмечались выраженные мнестико-интеллектуальные расстройства на фоне хронической гипоксемии, снижение критики к собственному состоянию. При осмотре - отеки передней брюшной стенки, голеней, стоп, артериальная гипертензия с повышением артериального давления до 180/100 мм рт.ст., снижение уровня насыщения крови кислородом ( $\left.\mathrm{SpO}_{2}\right)$ до 76\% в положении сидя.

По результатам проведенных лабораторных тестов выявлено снижение уровня витамина D до 7,3 нг/мл, остальные показатели (в том числе показатели липидного и углеводного обмена) были в пределах нормы.

По результатам респираторного мониторинга было зарегистрировано 129,4 эпизода апноэ обструктивного генеза и 14,6 эпизода апноэ центрального генеза. Индекс апноэ/гипопноэ составил 144 события в час. Максимальная продолжительность апноэ - 49 с; средняя длительность апноэ - 14 с. Нарушения дыхания сопровождались эпизодами тяжелых десатураций с минимальным значением 43\%. Средние показатели $\mathrm{SpO}_{2}$ в течение ночи составили $67 \%$ (рис. 1).

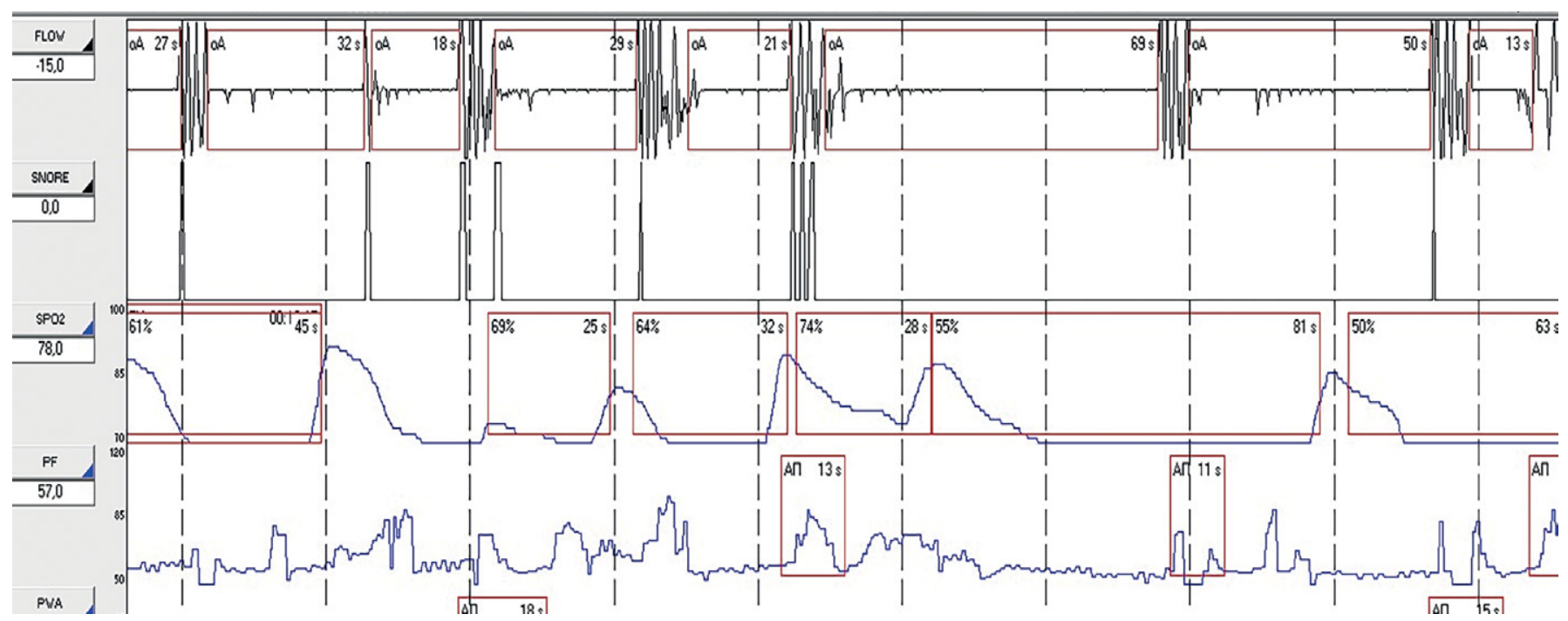

Рисунок 1. Респираторный мониторинг (5-минутная развертка). 
По результатам спирометрии было выявлено значительное снижение жизненной емкости легких, умеренное снижение бронхиальной проходимости по бронхам мелкого и среднего калибра смешанного генеза (обструкция с рестрикцией).

По результатам проведенного обследования был установлен диагноз «Морбидное ожирение 3 ст. Сверхожирение. Артериальная гипертензия 2 ст. Стеатогепатоз. Язвенная болезнь 12-перстной кишки, впервые выявленная. Язвы (2) луковицы 12-перстной кишки. Синдром апноэ сна смешанного генеза крайне тяжелой степени. Синдром альвеолярной гиповентиляции. Хроническая ночная гипоксемия крайне тяжелой степени».

С учетом тяжести выявленных респираторных нарушений, крайне высокого риска развития периоперационных осложнений на фоне хронической гипоксемии (в том числе, высокого риска развития несостоятельности желудочного шва, гнойно-воспалительного процесса в области вмешательства), крайне низкой комплаентности пациентки было принято решение о попытке проведения комплексной предоперационной подготовки в условиях хирургического отделения.

Программа предоперационной подготовки включала диетотерапию (1000 ккал/сут), антисекреторную, эрадикационную, гипотензивную, противоотечную терапию, профилактику тромбоэмболических осложнений, ингаляции с бронхолитиками, BiPAР-терапию (BiLevel (Biphasic) Positive Airway Pressure).

После проведения ВіРАР-терапии в течение 3 сут с целью контроля эффективности лечения была выполнена мониторная пульсоксиметрия; отмечено сокращение числа десатураций (индекс десатураций (ИД) составил 26,3 соб./час), однако сохранялась гипоксемия крайне тяжелой степени (средняя сатурация в течение ночи сохранялась на уровне 69,57\%, минимальная сатурация составила 51\%). Было принято решение о дополнении ВіРАР-терапии оксигенотерапией со скоростью потока увлажненного кислорода 4 л/мин. Во время бодрствования пациентки оксигенотерапия не проводилась (рис. 2).

При контрольной пульсоксиметрии через 2 сут отмечено ухудшение картины в виде нарастания ИД до 35,1 соб./час, сохранения показателей средней сатурации в течение ночи на уровне $72 \%$, базовая сатурация составила 73,66\%, минимальная сатурация - 51\%; клинически отмечено ухудшение состояния в виде появления жалоб на головные боли, нарастания общей слабости (рис. 3).

В связи с отсутствием значимой положительной динамики на фоне проводившегося лечения было высказано предположение о развитии у пациентки так называемого гипоксического стимула. Была проведена коррекция параметров оксигенотерапии - поток кислорода был уменьшен до 2 л/мин.

По данным контрольной пульсоксиметрии отмечена положительная динамика - отмечено повышение средней сатурации в течение ночи до 78,84\%, базовая сатурация составила $82,16 \%$, минимальная сатурация $58 \%$ (рис. 4).

На этом фоне отмечено субъективное улучшение состояния в виде уменьшения явлений общей слабости, выраженности одышки, исчезновения головной боли, стабилизации показателей артериального давления, повышения физической активности пациентки (до начала предоперационной подготовки пациентка перемещалась только в пределах палаты с помощью костылей; после предоперационной подготовки пациентка была активна в пределах отделения, перемещалась без костылей). По данным контрольной эзофагогастродуоденоскопии отмечено полное рубцевание ранее выявленных язв луковицы 12-перстной кишки.

Был проведен консилиум с участием хирургов, терапевта, сомнолога, анестезиолога-реаниматолога: с учетом улучшения клинико-инструментальной картины на фоне предоперационной подготовки, нецелесообразности дальнейшей пролонгации предоперационной подготовки, бесперспективности продолжения консервативного лечения ожирения пациентка может быть оперирована в плановом порядке, однако риск развития периоперационных осложнений (в т.ч. кардиальных и респираторных) крайне высок.

Длительность предоперационной подготовки составила 19 сут. Отмечено снижение МТ на 40 кг (\% потери массы тела 17,4). Бо́льшая часть потерянной массы тела в представленном клиническом случае - результат массивной противоотечной терапии. Также большую роль сыграли повышение уровня оксигенации крови и нормализация сна; у пациентов с СОАС отмечается выпадение глубокой фазы сна и, как следствие, нарушение выработки ряда гормонов (в частности, соматотропного гормона) и развитие инсулинорезистентности. При нормализации оксигенации крови у таких пациентов отмечается снижение МТ.

В плановом порядке пациентка была оперирована в объеме лапароскопической рукавной резекции желудка по стандартной методике с применением низкоопиоидной анестезии. Проведена экстубация на операционном столе через 5 мин после окончания операции, после чего пациентка была переведена в отделение реанимации. Сразу после экстубации была продолжена ВіРАР-терапия в сочетании с оксигенотерапией. Через 2 ч после операции было разрешено пить воду, через 6 ч после операции пациентка была активизирована в условиях отделения реанимации. Через 17 ч после оперативного лечения - перевод в профильное отделение.

Течение послеоперационного периода без осложнений. В связи с проживанием пациентки в другом регионе послеоперационное наблюдение было пролонгировано до 6 сут, после чего была осуществлена выписка из стационара. После выписки из стационара было рекомендовано продолжить терапию низкомолекулярными гепаринами в течение 3 нед. Также пациентке было рекомендовано продолжение ВіРАР-терапии амбулаторно, однако от данного вида лечения пациентка отказалась ввиду высокой стоимости лечебного оборудования.

При динамическом наблюдении через 1 год после операции отмечены снижение МТ до 153 кг (\% потери массы тела 33,5), нормализация показателей артериального давления с отменой гипотензивных препаратов. Отмечено значительное улучшение качества жизни: по опроснику оценки качества жизни SF-36 повышение показателя физического здоровья (PH) с 17,07 до 49,3, показателя психологического здоровья (МН) - с 17,87 до 60,84. 


\begin{tabular}{|c|c|c|c|c|c|c|c|}
\hline \multicolumn{2}{|c|}{ Recording info } & Hrs. & Min. & Sp02 Summary & $\%$ & Pulse summary & PR (bpm) \\
\hline \multirow{2}{*}{\multicolumn{2}{|c|}{$\begin{array}{l}\text { Recording time } \\
\text { Undefined/Motion }\end{array}$}} & 4 & 49 & Highest SpO2 & 99 & Highest Pulse rate & 121 \\
\hline & & 3 & 201.5 & Lowest SpO2 & 51 & Lowest Pulse rate & \multirow{2}{*}{$\begin{array}{r}41 \\
95.7 \\
\end{array}$} \\
\hline \multicolumn{2}{|c|}{ Net time } & 1 & 27.5 & Mean SpO2 & 69,57 & Mean Pulse rate & \\
\hline & & & & \multicolumn{4}{|c|}{ DeSat summary } \\
\hline $\begin{array}{c}\text { SpO2 } \\
\text { distribution }\end{array}$ & Min. & $\%$ time & \#DeSats & & & Total number & \multirow{2}{*}{$\begin{array}{c}67 \\
26.3 \\
70.86 \\
\end{array}$} \\
\hline $100 \%-94 \%$ & 3.3 & 2.2 & 0 & & & Baseline SpO2 (\%) & \\
\hline $93 \%-88 \%$ & 2.4 & 1.6 & 0 & & & & \\
\hline $87 \%-80 \%$ & 2.9 & 1.9 & 0 & & & Sp02 below $89 \%$ & Time \\
\hline $79 \%-70 \%$ & 39.1 & 25.6 & 17 & & & Max continuous period & \\
\hline $69 \%-60 \%$ & 33.1 & 21.7 & 30 & & & Total time DeSat 89 & 54 \\
\hline $59 \%-50 \%$ & 14.9 & 9.8 & 20 & & & Total time SpO2 89 & 283 \\
\hline $49 \%-40 \%$ & 0 & 0 & 0 & & & & \\
\hline & & & & & & $\begin{array}{l}\text { Motion summary } \\
\text { Motion }\end{array}$ & $\begin{array}{cc}\text { Min. } & \text { \% time } \\
65.1 & 22.5\end{array}$ \\
\hline
\end{tabular}

Рисунок 2. Мониторная пульсоксиметрия (3-и сутки ВіРАР-терапии).

\begin{tabular}{|c|c|c|c|c|}
\hline Recording info & Hrs. & Min. & SpO2 Summary & $\%$ \\
\hline Recording time & 2 & 19 & Highest SpO2 & 94 \\
\hline Undefined / Motion & 1 & 99 & Lowest SpO2 & 51 \\
\hline Net time & 0 & 40 & Mean SpO2 & 72 \\
\hline
\end{tabular}

\begin{tabular}{lc}
\hline \multicolumn{1}{c}{ Pulse summary } & PR (bpm) \\
\hline Highest Pulse rate & 127 \\
Lowest Pulse rate & 62 \\
Mean Pulse rate & 96.2 \\
\hline
\end{tabular}

\begin{tabular}{cccc}
\hline $\begin{array}{c}\text { SpO2 } \\
\text { distribution }\end{array}$ & Min. & \% time & \#DeSats \\
\hline $100 \%-94 \%$ & 0.8 & 1.4 & 0 \\
$93 \%-88 \%$ & 1.8 & 3.1 & 0 \\
$87 \%-80 \%$ & 1 & 1.6 & 0 \\
$79 \%-70 \%$ & 29.2 & 50.2 & 12 \\
$69 \%-60 \%$ & 5.2 & 9 & 15 \\
$59 \%-50 \%$ & 3.4 & 5.8 & 7 \\
$49 \%-40 \%$ & 0 & 0 & 0 \\
\hline
\end{tabular}

\begin{tabular}{|c|c|c|}
\hline \multicolumn{3}{|l|}{ DeSat summary } \\
\hline Total number & & 34 \\
\hline O2 DeSaturation Index DI & & 5.1 \\
\hline Baseline SpO2 (\%) & & 3.66 \\
\hline Sp02 below $89 \%$ & Min. & Time \\
\hline Max continuous period & 38.3 & $23: 40: 21$ \\
\hline Total time DeSat 89 & 26 & \\
\hline Total time SpO2 89 & 136 & \\
\hline Motion summary & Min. & $\%$ time \\
\hline Motion & 18.2 & 13.1 \\
\hline
\end{tabular}

Рисунок 3. Мониторная пульсоксиметрия (6-е сутки ВіРАР-терапии).

\begin{tabular}{|c|c|c|c|}
\hline \multicolumn{2}{|c|}{ Recording info } & Hrs. & Min. \\
\hline \multicolumn{2}{|c|}{ Recording time } & 2 & 14 \\
\hline \multicolumn{2}{|c|}{ Undefined / Motion } & 0 & 5 \\
\hline \multicolumn{2}{|c|}{ Net time } & 2 & 9 \\
\hline $\begin{array}{c}\mathrm{SpO2} \\
\text { distribution }\end{array}$ & Min. & $\%$ time & \#DeSats \\
\hline $100 \%-94 \%$ & 0.7 & 0.5 & 0 \\
\hline $93 \%-88 \%$ & 1.9 & 1.4 & 0 \\
\hline $87 \%-80 \%$ & 52.5 & 39.3 & 14 \\
\hline $79 \%-70 \%$ & 76.2 & 57 & 116 \\
\hline $69 \%-60 \%$ & 1.8 & 1.3 & 3 \\
\hline $59 \%-50 \%$ & 0.3 & 0.2 & 1 \\
\hline $49 \%-40 \%$ & 0 & 0 & 0 \\
\hline
\end{tabular}

\begin{tabular}{lc}
\hline \multicolumn{1}{c}{ SpO2 Summary } & $\%$ \\
\hline Highest $\mathrm{SpO} 2$ & 99 \\
Lowest $\mathrm{SpO} 2$ & 58 \\
Mean SpO2 & 78.84 \\
\hline
\end{tabular}

\begin{tabular}{lc}
\hline \multicolumn{1}{c}{ Pulse summary } & PR (bpm) \\
\hline Highest Pulse rate & 107 \\
Lowest Pulse rate & 40 \\
Mean Pulse rate & 84.2 \\
\hline
\end{tabular}

\begin{tabular}{lc}
\hline \multicolumn{1}{c}{ DeSat summary } & \\
\hline Total number & 134 \\
O2 DeSaturation Index DI & 60.1 \\
Baseline SpO2 (\%) & 82.16 \\
\hline
\end{tabular}

\begin{tabular}{lcc}
\hline \multicolumn{1}{c}{ Sp02 below 89\% } & Min. & Time \\
\hline Max continuous period & 69 & $21: 53: 18$ \\
Total time DeSat 89 & 95 & \\
Total time SpO2 89 & 132 & \\
\hline
\end{tabular}

\begin{tabular}{ccc}
\hline Motion summary & Min. & \% time \\
\hline Motion & 4.7 & 3.5 \\
\hline
\end{tabular}

Рисунок 4. Мониторная пульсоксиметрия (на фоне ВіРАР-терапии и низкопоточной оксигенотерапии). 


\section{ОБСУЖДЕНИЕ}

Объем и продолжительность предоперационной подготовки пациентов с морбидным ожирением в сочетании с СОАС тяжелой степени до сих пор остаются дискутабельными.

Стандартная методика включает в себя диетотерапию, подбор терапии для коррекции коморбидной патологии, подбор параметров СРАР/ВіРАР-терапии с целью коррекции апноэ, имплантацию интрагастрального баллона. Предоперационная подготовка проводится амбулаторно, длится от нескольких недель до нескольких месяцев, а оперативное лечение проводится только после стабилизации МТ и компенсации коморбидных состояний. Данная методика имеет ряд отрицательных моментов, обусловленных невозможностью адекватного контроля эффективности проводимой терапии и трудностями ее коррекции, высокой стоимостью оборудования для проведения амбулаторной СРАР-терапии (как и в случае установки внутрижелудочного баллона перед операцией), а также повышением риска осложнений периоперационного периода. Так, одним из клинических проявлений СОАС является гастроэзофагеальный рефлюкс в период сна; после установки внутрижелудочного баллона отмечается высокая частота развития тошноты и рвоты; в совокупности эти два фактора повышают риск развития респираторных осложнений на этапе предоперационной подготовки из-за более высокой вероятности аспирации желудочного содержимого. Длительность предоперационной подготовки также является отрицательным моментом из-за низкой комплаентности данной категории пациентов. Так, в период с 2011 по 2016 гг. 25 пациентов отказались от предложенного плана дооперационного лечения из-за его продолжительности и стоимости лечебного оборудования; также был зафиксирован 1 летальный исход у пациента с ожирением 3 ст. и СОАС тяжелой степени в первые сутки после имплантации внутрижелудочного баллона вследствие развития аспирации желудочного содержимого.

Разработанная методика подразумевает проведение краткосрочной комплексной предоперационной подготовки в условиях стационара, а также отказ от имплантации внутрижелудочного баллона. Программа включает в себя диетотерапию с суточной калорийностью рациона 1000 ккал, лечение сопутствующих заболеваний, инициацию СРАР/ВіРАР-терапии, при необходимости - дополнительное проведение оксигенотерапии. В отличие от стандартной методики продолжительность ускоренной предоперационной подготовки составляет от нескольких суток до нескольких недель. К настоящему времени по указанной ускоренной методике нами подготовлены, а затем прооперированы 63 пациента с мор- бидным ожирением и СОАС тяжелой степени. При этом не было зафиксировано каких-либо осложнений периоперационного периода, связанных с ее применением; летальных исходов не было. Представленное наблюдение демонстрирует возможности применения современных методик ускоренной реабилитации для снижения операционного риска при подготовке крайне тяжелых пациентов с СОАС к выполнению бариатрических операций.

\section{ЗАКЛЮЧЕНИЕ}

Программа ускоренной периоперационной реабилитации может быть использована как альтернатива стандартной программе реабилитации с имплантацией интрагастрального баллона у пациентов с морбидным ожирением в сочетании с СОАС. Применение данной методики позволяет повысить эффективность лечения данной категории пациентов, а также снизить риск развития периоперационных осложнений. Тем не менее для полноценного анализа требуется накопление опыта и большее количество пролеченных пациентов с использованием описанной программы ускоренной предоперационной реабилитации, в связи с чем полученные результаты, описанные в данном клиническом наблюдении, требуют острожной интерпретации.

\section{ДОПОЛНИТЕЛЬНАЯ ИНФОРМАЦИЯ}

Источник финансирования. Обследование пациентки проводилось на базе ФГАУ «НМИЦ «Лечебно-реабилитационный центр» Минздрава России без привлечения дополнительного финансирования.

Согласие пациента. Пациент добровольно подписал информированное согласие на публикацию персональной медицинской информации в обезличенной форме в журнале «Ожирение и метаболизм».

Конфликт интересов. Авторы декларируют отсутствие явных и потенциальных конфликтов интересов, связанных с содержанием настоящей статьи.

Участие авторов. Орловская Е.С. - получение, анализ данных и интерпретация результатов; написание статьи; Ачкасов Е.Е. - существенный вклад в разработку дизайна исследования; внесение в рукопись важных правок с целью повышения научной ценности статьи; Яшков Ю.И. существенный вклад в разработку дизайна исследования; внесение в рукопись важных правок с целью повышения научной ценности статьи; Зорин Е.А. - существенный вклад в разработку дизайна исследования; внесение в рукопись важных правок с целью повышения научной ценности статьи; Ермаков Н.А. - существенный вклад в разработку дизайна исследования; внесение в рукопись важныхправок с целью повышения научной ценности статьи. Все авторы одобрили финальную версию статьи перед публикацией, выразили согласие нести ответственность за все аспекты работы, подразумевающую надлежащее изучение и решение вопросов, связанных с точностью или добросовестностью любой части работы.

\section{СПИСОК ЛИТЕРАТУРЫ | REFERENCES}

Dietz WH, Baur LA, Hall K, et al. Management of obesity: improvement of health-care training and systems for prevention and care. Lancet. 2015;385:2521-2533. doi: https://doi.org/10.1016/S0140-6736(14)61748-7

2. Kelly T, Yang W, Chen CS, et al. Global burden of obesity in 2005 and projections to 2030. Int J Obes (Lond). 2008;32:1431-1437. doi: https://doi.org/10.1038/ijo.2008.102

3. Hakim NS, Favretti F, Segato G, Dillemans B. Bariatric surgery. UK; 2011
4. SAGES guideline for clinical application of laparoscopic bariatric surgery. Surg Obes Relat Dis. 2009;5(3):387-405. doi: https://doi.org/10.1016/j.soard.2009.01.010

5. Lemanu DP, Singh PP, Berridge K, et al. Randomized clinical trial of enhanced recovery versus standard care after laparoscopic sleeve gastrectomy. Br J Surg. 2013;100:482-489. doi: https://doi.org/ 10.1002/bjs.9026 
6. Joliat GR, Labgaa I, Petermann D, et al. Cost-benefit analysis of an enhanced recovery protocol for pancreaticoduodenectomy. Br J Surg. 2015;102:1676-1683. doi: https://doi.org/10.1002/bjs.9957

7. King PM, Blazeby JM, Ewings P, et al. The influence of an enhanced recovery programme on clinical outcomes, costs and quality of life after surgery for colorectal cancer. Colorectal Dis. 2006;8:506-513. doi: https://doi.org/10.1111/j.1463-1318.2006.00963.x

8. Thorell A, MacCormick AD, Awad $S$, et al. Guidelines for perioperative care in bariatric surgery: enhanced recovery after surgery (ERAS) society recommendations. World J Surg. 2016;40:2065-2083. doi: https://doi.org/10.1007/s00268-016-3492-3
9. Vasu TS, Grewal R, Doghramji K. Obstructive sleep apnea syndrome and perioperative complications: a systematic review of the literature. J Clin Sleep Med. 2012:8:199-207. doi: https://doi.org/10.5664/jcsm.1784

10. Kushida CA, Littner MR, Hirshkowitz M, et al. Practice Parameters for the Use of Continuous and Bilevel Positive Airway Pressure Devices to Treat Adult Patients With Sleep-Related Breathing Disorders. Sleep 2006;29(3):375-380. doi: https://doi.org/10.1093/sleep/29.3.375

11. Lemanu DP, Srinivasa S, Singh PP, et al. Optimizing perioperative care in bariatric surgery patients. Obes Surg. 2012;22:979-990. doi: https://doi.org/10.1007/s11695-012-0648-6

\section{ИНФОРМАЦИЯ ОБ АВТОРАХ [AUTHORS INFO]:}

*Орловская Екатерина Сергеевна [Ekaterina S. Orlovskaya, MD]; адрес: Россия, 125367, Москва, Иваньковское шоссе, д. 3 [address: 3 Ivan'kovskoe Highway, 125367 Moscow, Russia]; ORCID: https://orcid.org/0000-0003-0871-3067; eLibrary SPIN: 8336-0910; e-mail: eka2057@mail.ru

Ачкасов Евгений Евгеньевич, Д.М.Н., професcop [Evgeniy E. Achkasov, MD, PhD, Professor]; ORCID: https://orcid.org/0000-0001-9964-5199; eLibrary SPIN: 5291-0906; e-mail: 2215.g23@rambler.ru Яшков Юрий Иванович, д.м.н. [Yury I. Yashkov, MD, PhD]; ORCID: https://orcid.org/0000-0001-6592-1036; eLibrary SPIN: 8933-3745; e-mail: yu@yashkov.ru

Зорин Евгений Александрович, к.M.H. [Evgeniy A. Zorin, MD, PhD]; ORCID https://orcid.org/0000-0002-0624-540X; e-mail: zorin.e.al@gmail.com

Ермаков Николай Александрович, к.м.н. [Nikolay A. Ermakov, MD, PhD]; ORCID: https://orcid.org/0000-0002-1271-7960; eLibrary SPIN: 5985-9032; e-mail: n-ermakov@yandex.ru

*Автор, ответственный за переписку/ Corresponding author.

\section{ЦИТИРОВАТЬ:}

Орловская Е.С., Ачкасов Е.Е., Яшков Ю.И., Зорин Е.А., Ермаков Н.А. Ускоренная предоперационная подготовка В составе комплексной периоперационной реабилитации пациентов со сверхожирением и синдромом обструктивного апноэ (клиническое наблюдение) // Ожирение и метаболизм. — 2021. — Т. 18. — №4. — С. $432-437$. doi: https://doi.org/10.14341/omet12724

\section{TO CITE THIS ARTICLE:}

Orlovskaya ES, Achkasov EE, Yashkov Yul, Zorin EA, Ermakov NA. Enchanced preoperative rehabilitation as part of the complex perioperative rehabilitation of patients with super-obesity and obstructive sleep apnea syndrome (clinical outlook). Obesity and metabolism. 2021;18(4):432-437. doi: https://doi.org/10.14341/omet12724 